\title{
Effects of complementary grazing by goats on sward composition and on sheep performance managed during lactation in perennial ryegrass and white clover pastures
}

\author{
M. del Pozo * , K. Osoro, R. Celaya \\ CIATA, Apdo 13, 33300 Villaviciosa, Asturias, Spain
}

Accepted 15 September 1997

\begin{abstract}
The liveweight (LW) response of Gallega and Lacha ewes with their single suckling lambs when grazing with a mob of goats on perennial ryegrass (Lolium perenne L.)/white clover (Trifolium repens L.) pastures and its consequence on sward composition were analysed. From early March (turnout) to mid-June (lamb weaning), swards were grazed either simultaneously mixed in a 1:1 goat to sheep ratio or separately in a goat-first and sheep-last sequential grazing at 6 or $8 \mathrm{~cm}$ sward heights or in a 4-paddock rotation where goats grazed swards from 9 to $7 \mathrm{~cm}$ followed by sheep from 7 to $4 \mathrm{~cm}$. Changes in botanical composition and in sheep liveweight performance were more significantly affected by the management and species grazing than by the sward height treatment. Swards where goats were last in had higher herbage masses, higher live clover and lower dead and stem proportions than swards where sheep and goats were mixed or sheep were last in. As a consequence of a sward clover enhancement over all treatments, ewe and lamb performances were benefited especially in the Lacha genotype, with the rotational and sequential managements having, respectively, an output of 298 and $252 \mathrm{~kg} \mathrm{LW} / \mathrm{ha}$ greater than the mixed management. These results suggest that goats could be used as a pasture management tool when integrated with sheep under these different grazing systems to enhance ovine performance during lactation. (C) 1998 Elsevier Science B.V. All rights reserved.
\end{abstract}

Keywords: Sheep; Goat; Clover; Grazing management; Performance

\section{Introduction}

Based on reports performed from temperate grasslands (Clark et al., 1982; Russel et al., 1983; Grant et al., 1984), it appears that goats might be used to improve pastures for sheep production, particularly

\footnotetext{
* Corresponding author.
}

in grass / clover swards. When goats are used exclusively, the swards tend to contain more clover than grazed by sheep (Penning et al., 1996). Using goats to increase the proportion of clover in the sward could increase the supply of high-quality feed for growing weaned lambs as it has been reported in UK by del Pozo et al. (1996), but further work with suckling lambs and lactating ewes has only been carried out in the southern hemisphere by Townsend and Radcliffe (1990) and Radcliffe et al. (1991). 
Goats require careful management in sown swards, as sward surface heights should be above $6 \mathrm{~cm}$ to avoid suppression of intake (Merchant and Riach, 1994). Then, managing grazing systems with breeding does and sheep will depend on the degree of competition between these species, and could involve alternative sward height managements during the grazing cycle. Osoro and Martinez (1995), working in the North of Spain, used a mixture of feral goats and native sheep to improve pasture quality and profitability of marginal areas. Sheep have also been shown to benefit when integrated with fibreproducing goats in a concurrent mixed grazing (Collins, 1989; Townsend and Radcliffe, 1990; Radcliffe et al., 1991) or separate grazing at different times on the same pasture (del Pozo et al., 1996; Bown et al., 1989). However, the suitability of these management alternatives in temperate pastures of Southern European countries is not yet defined, and managerial decisions would be better achieved if sward conditions are carefully characterized.

Therefore, the objective of this study was to examine the feasibility of sheep and goat cograzing to increase clover persistence in the sward, and improve ovine performance under rotational, sequential or mixed managements maintained at two different herbage allowances.

\section{Materials and methods}

\subsection{Site}

The current experiment was conducted in 1995 at La Mata Research Farm, Grado, located at $50 \mathrm{~m}$ a.s.l, in Asturias, North of Spain. Total annual precipitation averages $1032 \mathrm{~mm}$ (917-1380 mm range) and temperature averages $13.2^{\circ} \mathrm{C} \quad\left(10.1-15.2^{\circ} \mathrm{C}\right.$ range) with June, July, August and September having highest daily temperatures and lowest total rainfall amounts of the year. In November 1991, a mixture of $20 \mathrm{~kg} / \mathrm{ha}$ of perennial ryegrass (Lolium perenne L. cv. Phoenix) plus $3 \mathrm{~kg} /$ ha of white clover (Trifolium repens L. cv. Huia) were sod seeded in the experimental area. On 25 January of the experimental year, the sward received a unique application of $40 \mathrm{~kg} \mathrm{~N} / \mathrm{ha}, 100 \mathrm{~kg} \mathrm{P}_{2} \mathrm{O}_{5} / \mathrm{ha}$ and 80 kg $\mathrm{K}_{2} \mathrm{O} /$ ha.

\subsection{Design}

From March 7 to June 16, 10 pastures of 0.7 -ha size were grazed with a herd of 54 dry Cashmere goats plus 48 autochthonous goats $(30.8 \pm 0.34$ and $45.3 \pm 0.47 \mathrm{~kg}$ of initial adult $\mathrm{LW}$, respectively) and 42 Gallega plus 66 Lacha ewes $(28.3 \pm 0.32$ and $32.0 \pm 0.52 \mathrm{~kg}$ of initial adult $\mathrm{LW}$, respectively) rearing their single lambs $(10.8 \pm 0.45$ and $11.2 \pm$ $0.41 \mathrm{~kg}$ of initial LW). Six paddocks were either continuously grazed under a first (goats)-last (sheep) sequential grazing or under a mixed grazing with a stock equivalence on a per head basis of 1:1 at two different available sward heights of $6 \mathrm{~cm}$ (Low) or 8 $\mathrm{cm}$ (High), whereas the remainder four paddocks were managed in a rotational management where sheep grazed swards from 7 to $4 \mathrm{~cm}$ (Low) after being previously grazed by goats from 9 to $7 \mathrm{~cm}$ (High). The different management and height treatments were randomized into the trial area and grazed by 'core' (tester) groups of sheep and goats balanced as far as possible on initial lamb liveweight and birth date, and on doe and lactating ewe breeds. Additional 'put and take' (replacement) ewes and/or goats were introduced or withdrawn to control mean sward heights.

\subsection{Management}

Suckling lambs born in January/February grazed with their dams from turnout until weaning. Within a sequential treatment, tester plus replacement sheep were alternated with goats between paddocks in a continuous succession of a 2-paddock system and that occurred simultaneously with the beginning of each rotation cycle in the rotational treatment on 10 April, 15 May and 16 June. Foot rot problems were controlled by using a zinc sulphate footbath, and individual animals were regularly drenched with $\mathrm{Ne}$ tobimín (200 mg/10 kg LW) to suppress internal parasites.

\subsection{Measurements}

\subsubsection{Pasture}

Air temperature and total rainfall observations were made each day by meteorological equipment located at the experimental site. Pasture height was 
measured weekly with a sward stick from the Hill Farming Research Organisation (Barthram, 1986). Fifty observations of the undisturbed sward surface height were made at random per paddock. Frequencies of sward heights were fitted for each treatment following the Gibb and Ridout (1986) procedure to estimate the proportion of the shorter ('frequently') and the taller ('infrequently') grazed sample populations.

The frequency of distribution of the different herbage components within the sward canopy was determined at the beginning ( 7 March), at the end of the experiment (16 June) and whenever animal species end a rotational and sequential cycle (10 April and 15 May) by recording stratified contacts in 25 points taken at random per paddock with an inclined $\left(32.5^{\circ}\right.$ ) point quadrat apparatus (Rhodes and Collins, 1993). As the point quadrat needle, mounted on a frame, passed through the sward each contact of herbage height was noted after ground zero correction and classed using the following morphological units: perennial ryegrass (leaf, pseudostem, flowerstem or dead), white clover (laminae-petiole, stolon, flower or dead), other species and herbaceous weeds. Simultaneously to the assessments on canopy composition, herbage mass was monitored by cutting to ground level ten strips $(1 \times 0.2 \mathrm{~m})$ per paddock. Samples were subsequently washed and oven-dried overnight at $80^{\circ} \mathrm{C}$ to determine dry matter content.

\subsubsection{Stock}

Ewe-liveweight changes were monitored by weighing of tester animals to the nearest $0.1 \mathrm{~kg}$ at the beginning of the experiment, at the end of the grazing season, and on each day that sward composition was determined. Body conditions were scored in ewes by the same operator and followed the 5-point scale used by Russel et al. (1969). Lambs were weighed at 1 day of age and each time that their dams were weighed. To determine overall animal production per hectare, sheep and goat stocking rates were recorded weekly having in account the animals added to or removed from paddocks to control sward height as they were weighed on and off the paddocks. Grab faecal samples were taken from all core groups of does and ewes at the beginning and end of the grazing season, and analysed immediately for faecal parasite egg concentration.

\subsection{Statistical analysis}

Analysis of variance was used to examine the effect of treatments on liveweight and sward composition changes occurred over the experiment by running the Genstat 5.2. statistical package (Lawes Agricultural Trust, 1990). Estimates of the variability on ewe and lamb performances were derived from the variation of the tester animals occurred within each paddock with data being analysed by a split-plot design, where animal units within a paddock were considered as blocks and grazing management $X$ sward height $\times$ sampling date as the treatment model. Effects of treatment and sampling date were examined in herbage variables using the variance between paddocks and between sampling date as the error treatment. Faecal egg counts and the count of herbage contacts were transformed by $\log _{10}+1$ before analysis, but only back-transformed values expressed as proportions are included in the text. A Pearson's $\chi^{2}$ was used to describe the pattern of height distribution by comparing across treatments the proportion of measurements falling above $10 \mathrm{~cm}$.

When variability of sheep performance was considered between management and height treatments, the data were not fully balanced. Then, overall differences that occurred between grazing managements and between height treatments within a grazing management were analysed separately for each sampling period and their level of significance determined by using Student's paired $t$-test comparisons with pooled estimates of the standard errors of difference of means (SED). Differences in sward composition due to species grazing were analysed in a similar manner as mean values were determined separately for each treatment in paddocks last grazed by goats, sheep or both species.

\section{Results}

\subsection{Weather}

The weather was abnormally dry as only 181.6 $\mathrm{mm}$ of rainfall occurred over the trial period. However, temperature values were average, and monthly means increased during the trial from $11^{\circ} \mathrm{C}$ in March till $17.2^{\circ} \mathrm{C}$ in June. 
Table 1

Mean herbage mass ( $\mathrm{kg} \mathrm{DM} / \mathrm{ha}$ ) at each sampling date and proportion of sward height measurements falling above $10 \mathrm{~cm}$ in the sward across the different treatments during the whole experimental period

\begin{tabular}{|c|c|c|c|c|c|c|c|c|c|c|}
\hline \multirow[t]{4}{*}{ Date } & \multicolumn{9}{|c|}{ Grazing management } & \multirow[t]{4}{*}{$P^{1}$} \\
\hline & \multicolumn{3}{|l|}{ Rotational } & \multicolumn{3}{|l|}{ Sequential } & \multicolumn{3}{|l|}{ Mixed } & \\
\hline & \multicolumn{3}{|c|}{ Sward height } & \multirow[b]{2}{*}{ High } & \multirow[b]{2}{*}{ Low } & \multirow[b]{2}{*}{ SEM } & \multirow[b]{2}{*}{ High } & \multirow[b]{2}{*}{ Low } & \multirow[b]{2}{*}{ SEM } & \\
\hline & High & Low & SEM & & & & & & & \\
\hline \multicolumn{11}{|c|}{ Pasture mass $(n=10)$} \\
\hline 7 March & 22.39 & $2149^{\mathrm{a}}$ & 192.3 & $2301^{\mathrm{a}}$ & $2189^{\mathrm{a}}$ & 198.1 & $2289^{\mathrm{a}}$ & $2400^{\mathrm{a}}$ & 272.7 & NS \\
\hline 10 April & $2099^{\mathrm{a}}$ & $1532^{\mathrm{b}}$ & 250.0 & $2197^{a}$ & $1792^{\mathrm{ab}}$ & 205.2 & $1700^{\mathrm{ab}}$ & $1489^{\mathrm{b}}$ & 253.1 & $P<0.01$ \\
\hline 15 May & $2382^{\mathrm{a}}$ & $1400^{\mathrm{b}}$ & 233.4 & $2299^{a}$ & $1900^{\mathrm{ab}}$ & 213.3 & $1778^{\mathrm{b}}$ & $1525^{\mathrm{b}}$ & 261.4 & $P<0.001$ \\
\hline 16 June & $2200^{\mathrm{a}}$ & $1458^{\mathrm{b}}$ & 236.6 & $2125^{\mathrm{a}}$ & $1839^{\mathrm{ab}}$ & 222.2 & $1612^{b}$ & $1449^{\mathrm{b}}$ & 243.6 & $P<0.001$ \\
\hline \multicolumn{11}{|c|}{ Sward height proportion $(n=800)$} \\
\hline 7 March-16 June & $0.02^{\mathrm{a}}$ & $0.01^{\mathrm{a}}$ & 0.013 & $0.12^{\mathrm{b}}$ & $0.05^{\mathrm{a}}$ & 0.010 & $0.07^{\mathrm{a}}$ & $0.03^{\mathrm{b}}$ & 0.021 & $P<0.05$ \\
\hline
\end{tabular}

Means in the same row with different superscripts differs significantly $(P<0.05)$.

${ }^{1}$ Goat vs. sheep last grazing, NS = non-significant. 
Table 2

Proportions of ryegrass leaf, ryegrass stem (pseudostem + flowerstem), dead ryegrass, live white clover (laminae + petiole + stolon + flower) and dead clover in the sward across the different treatments at each sampling date

\begin{tabular}{|c|c|c|c|c|c|c|c|c|c|c|c|}
\hline \multirow[t]{4}{*}{ Components } & \multirow[t]{4}{*}{ Date } & \multicolumn{9}{|c|}{ Grazing management } & \multirow[t]{4}{*}{$P^{1}$} \\
\hline & & \multicolumn{3}{|c|}{ Rotational } & \multicolumn{3}{|c|}{ Sequential } & \multicolumn{3}{|l|}{ Mixed } & \\
\hline & & \multicolumn{6}{|c|}{ Sward height } & \multirow[b]{2}{*}{ High } & \multirow[b]{2}{*}{ Low } & \multirow[b]{2}{*}{ SEM } & \\
\hline & & High & Low & SEM & High & Low & SEM & & & & \\
\hline \multirow[t]{4}{*}{ Ryegrass leaf } & 7 March & $0.67^{\mathrm{ab}}$ & $0.68^{\mathrm{ab}}$ & 0.053 & $0.58^{\mathrm{a}}$ & $0.55^{\mathrm{a}}$ & 0.051 & $0.73^{b}$ & $0.72^{\mathrm{b}}$ & 0.059 & NS \\
\hline & 10 April & $0.58^{\mathrm{ab}}$ & $0.29^{\mathrm{c}}$ & 0.048 & $0.60^{\mathrm{ab}}$ & $0.50^{\mathrm{b}}$ & 0.043 & $0.69^{\mathrm{a}}$ & $0.65^{\mathrm{ab}}$ & 0.042 & $P<0.05$ \\
\hline & 15 May & $0.55^{\mathrm{a}}$ & $0.53^{\mathrm{ab}}$ & 0.037 & $0.50^{\mathrm{ab}}$ & $0.40^{\mathrm{b}}$ & 0.047 & $0.72^{\mathrm{c}}$ & $0.70^{\mathrm{c}}$ & 0.043 & NS \\
\hline & 16 June & $0.60^{\mathrm{b}}$ & $0.43^{\mathrm{a}}$ & 0.054 & $0.44^{\mathrm{a}}$ & $0.41^{\mathrm{a}}$ & 0.052 & $0.57^{\mathrm{b}}$ & $0.64^{\mathrm{b}}$ & 0.051 & $P<0.05$ \\
\hline \multirow[t]{4}{*}{ Ryegrass stem } & 7 March & $0.13^{\mathrm{ab}}$ & $0.11^{\mathrm{ab}}$ & 0.018 & $0.12^{\mathrm{ab}}$ & $0.16^{\mathrm{b}}$ & 0.021 & $0.10^{\mathrm{a}}$ & $0.09^{\mathrm{a}}$ & 0.018 & NS \\
\hline & 10 April & $0.05^{\mathrm{a}}$ & $0.40^{\mathrm{c}}$ & 0.017 & $0.04^{\mathrm{a}}$ & $0.12^{\mathrm{b}}$ & 0.019 & $0.02^{\mathrm{a}}$ & $0.02^{\mathrm{a}}$ & 0.017 & $P<0.001$ \\
\hline & 15 May & $0.02^{\mathrm{a}}$ & $0.02^{\mathrm{a}}$ & 0.021 & $0.05^{\mathrm{a}}$ & $0.03^{\mathrm{a}}$ & 0.017 & $0.03^{\mathrm{a}}$ & $0.00^{\mathrm{a}}$ & 0.020 & $P<0.05$ \\
\hline & 16 June & $0.03^{\mathrm{a}}$ & $0.15^{\mathrm{b}}$ & 0.022 & $0.06^{\mathrm{a}}$ & $0.08^{\mathrm{ab}}$ & 0.016 & $0.15^{\mathrm{b}}$ & $0.13^{\mathrm{b}}$ & 0.022 & $P<0.001$ \\
\hline \multirow[t]{4}{*}{ Dead ryegrass } & 7 March & $0.16^{\mathrm{ab}}$ & $0.18^{\mathrm{ab}}$ & 0.024 & $0.18^{\mathrm{ab}}$ & $0.20^{\mathrm{b}}$ & 0.023 & $0.11^{\mathrm{a}}$ & $0.15^{\mathrm{ab}}$ & 0.022 & NS \\
\hline & 10 April & $0.04^{\mathrm{c}}$ & $0.12^{\mathrm{b}}$ & 0.021 & $0.17^{\mathrm{ab}}$ & $0.22^{\mathrm{a}}$ & 0.019 & $0.05^{\mathrm{c}}$ & $0.06^{\mathrm{c}}$ & 0.019 & $P<0.001$ \\
\hline & 15 May & $0.27^{\mathrm{a}}$ & $0.43^{b}$ & 0.020 & $0.25^{\mathrm{a}}$ & $0.29^{\mathrm{a}}$ & 0.020 & $0.17^{\mathrm{c}}$ & $0.24^{\mathrm{a}}$ & 0.020 & $P<0.001$ \\
\hline & 16 June & $0.09^{\mathrm{b}}$ & $0.25^{\mathrm{c}}$ & 0.019 & $0.32^{\mathrm{a}}$ & $0.35^{\mathrm{a}}$ & 0.018 & $0.12^{\mathrm{b}}$ & $0.13^{\mathrm{b}}$ & 0.021 & $P<0.001$ \\
\hline \multirow[t]{4}{*}{ Live clover } & 7 March & $0.04^{\mathrm{a}}$ & $0.03^{\mathrm{a}}$ & 0.017 & $0.07^{\mathrm{a}}$ & $0.05^{\mathrm{a}}$ & 0.015 & $0.04^{\mathrm{a}}$ & $0.03^{\mathrm{a}}$ & 0.013 & NS \\
\hline & 10 April & $0.33^{\mathrm{c}}$ & $0.19^{\mathrm{ab}}$ & 0.019 & $0.19^{\mathrm{ab}}$ & $0.14^{\mathrm{b}}$ & 0.020 & $0.23^{\mathrm{a}}$ & $0.25^{\mathrm{a}}$ & 0.019 & $P<0.001$ \\
\hline & 15 May & $0.16^{\mathrm{b}}$ & $0.02^{\mathrm{c}}$ & 0.018 & $0.18^{\mathrm{ab}}$ & $0.23^{\mathrm{a}}$ & 0.021 & $0.07^{\mathrm{c}}$ & $0.05^{\mathrm{c}}$ & 0.015 & $P<0.001$ \\
\hline & 16 June & $0.26^{\mathrm{c}}$ & $0.15^{\mathrm{ab}}$ & 0.017 & $0.18^{\mathrm{a}}$ & $0.12^{\mathrm{ab}}$ & 0.020 & $0.16^{\mathrm{ab}}$ & $0.10^{\mathrm{b}}$ & 0.016 & $P<0.001$ \\
\hline \multirow[t]{4}{*}{ Dead clover } & 7 March & $0.00^{\mathrm{a}}$ & $0.00^{\mathrm{a}}$ & 0.005 & $0.00^{\mathrm{a}}$ & $0.00^{\mathrm{a}}$ & 0.009 & $0.01^{\mathrm{a}}$ & $0.00^{\mathrm{a}}$ & 0.006 & NS \\
\hline & 10 April & $0.00^{\mathrm{a}}$ & $0.00^{\mathrm{a}}$ & 0.004 & $0.00^{\mathrm{a}}$ & $0.00^{\mathrm{a}}$ & 0.008 & $0.00^{\mathrm{a}}$ & $0.00^{\mathrm{a}}$ & 0.005 & NS \\
\hline & 15 May & $0.00^{\mathrm{a}}$ & $0.00^{\mathrm{a}}$ & 0.007 & $0.00^{\mathrm{a}}$ & $0.02^{\mathrm{b}}$ & 0.007 & $0.01^{\mathrm{a}}$ & $0.00^{\mathrm{a}}$ & 0.004 & NS \\
\hline & 16 June & $0.00^{\mathrm{a}}$ & $0.01^{\mathrm{a}}$ & 0.009 & $0.00^{\mathrm{a}}$ & $0.00^{\mathrm{a}}$ & 0.008 & $0.00^{\mathrm{a}}$ & $0.00^{\mathrm{a}}$ & 0.007 & NS \\
\hline
\end{tabular}

Means in the same row with different superscripts differs significantly $(P<0.05)$.

${ }^{1}$ Goat vs. sheep last grazing, NS $=$ non-significant. 


\section{March control}

Rotational
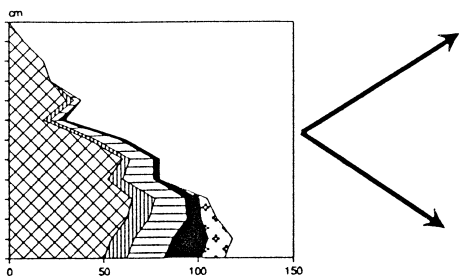

Sequential

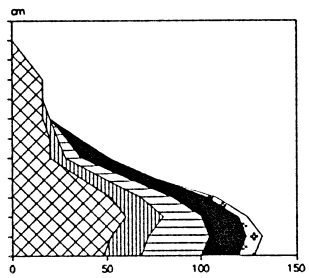

Mixed
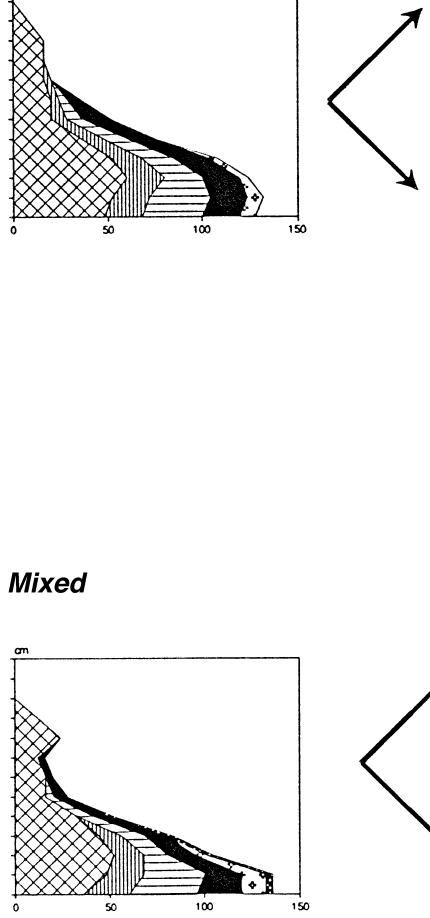

Low

(Sheep last grazing)

High

(Goat last grazing)

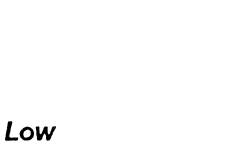

High
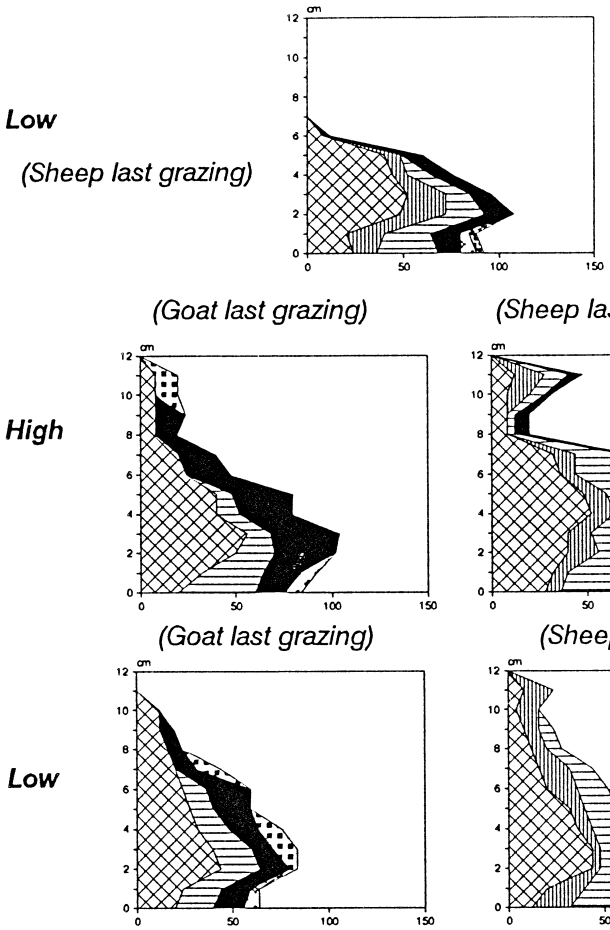

(Sheep last grazing)

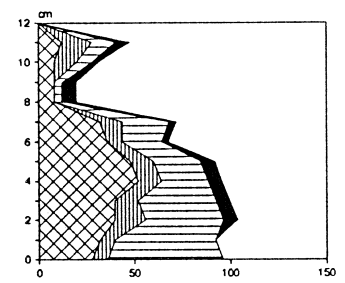

(Sheep last grazing)

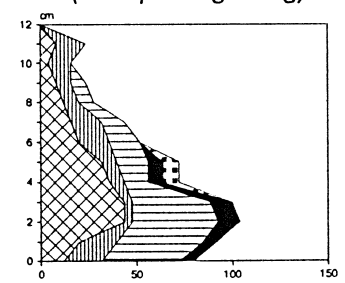

High

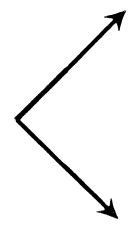

Low
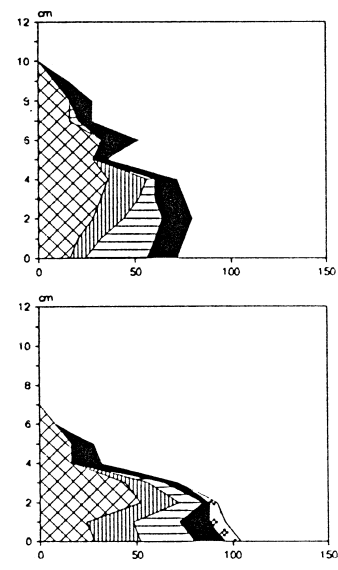

clover

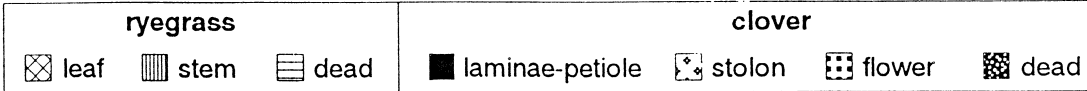


Table 3

Liveweight performance of the 'core' lambs suckling Lacha or Gallega ewes at each sampling date and grazing with goats in ryegrass / clover swards under different management and sward height treatments from turnout (7 March) to lamb weaning (16 June)

\begin{tabular}{|c|c|c|c|c|c|c|c|c|c|}
\hline & \multirow[t]{3}{*}{ Date } & \multicolumn{8}{|c|}{ Grazing management } \\
\hline & & \multicolumn{2}{|c|}{ Rotational } & \multicolumn{3}{|c|}{ Sequential } & \multicolumn{3}{|l|}{ Mixed } \\
\hline & & \multicolumn{5}{|c|}{ Sward height } & High & Low & SEM \\
\hline \multicolumn{10}{|l|}{ Lacha } \\
\hline \multirow[t]{2}{*}{ Liveweight (kg LW) } & 7 March & $10.9^{\mathrm{a}}$ & 1.72 & $11.2^{\mathrm{a}}$ & $11.6^{\mathrm{a}}$ & 1.62 & $10.8^{\mathrm{a}}$ & $11.7^{\mathrm{a}}$ & 1.99 \\
\hline & 16 June & $25.7^{\mathrm{a}}$ & 1.80 & $34.8^{\mathrm{b}}$ & $31.3^{\mathrm{c}}$ & 1.60 & $35.6^{\mathrm{b}}$ & $37.9^{\mathrm{b}}$ & 1.90 \\
\hline \multicolumn{10}{|l|}{ Gallega } \\
\hline \multirow[t]{3}{*}{ Liveweight (kg LW) } & 7 March & $10.5^{\mathrm{a}}$ & 1.79 & $10.4^{\mathrm{a}}$ & $10.7^{\mathrm{a}}$ & 1.58 & $11.1^{\mathrm{a}}$ & $11.3^{\mathrm{a}}$ & 1.91 \\
\hline & 10 April & $14.6^{\mathrm{a}}$ & 1.75 & $16.8^{\mathrm{ab}}$ & $18.9^{\mathrm{ab}}$ & 1.63 & $21.9^{\mathrm{b}}$ & $19.4^{\mathrm{ab}}$ & 1.87 \\
\hline & 15 May & $20.9^{\mathrm{a}}$ & 1.68 & $22.7^{\mathrm{ab}}$ & $25.5^{\mathrm{ab}}$ & 1.67 & $27.3^{\mathrm{b}}$ & $24.9^{\mathrm{ab}}$ & 2.01 \\
\hline
\end{tabular}

Means in the same row with different superscripts differs significantly $(P<0.05)$.

\subsection{Pasture}

Mean sward heights did not differ significantly between management treatments with $8.4,8.0$ and $8.1 \mathrm{~cm}$ being recorded in the rotational, sequential and mixed managements respectively of the high height treatments and 5.9, 6.3 and $6.1 \mathrm{~cm}$ being recorded in the rotational, sequential and mixed managements respectively of the low height treatments $(\mathrm{SED}=0.23)$. However, higher proportions of sward heights above $10 \mathrm{~cm}$ were found on the sequential treatment than on the rotational and mixed treatments $\left(\chi^{2}=6.93 ; d f=2 ; P<0.05\right)$. The amount of residual masses existing with goat last grazing was higher than with sheep last grazing at similar height treatments in the rotational and sequential managements $(P<0.01)$, and that was accompanied in both managements with differences in the distributions of heights, since paddocks last grazed from sheep had a more skewed distribution than paddocks last grazed from goats $\left(\chi^{2}=4.22 ; d f=1 ; P<0.05\right)$. As shown by Table 1 , the mixed management had fair residual mass values. Finally, the sequential and rotational managements resulted, in the high height treatments, in higher pasture masses than the mixed management.

Table 2 shows the proportions of the perennial ryegrass and white clover components recorded in each sward treatment at each sampling date, whereas Fig. 1 displays the different sward treatment profiles at the beginning and end of the experiment.

At the beginning of the grazing period (7 March), there were no significant differences on ryegrass and clover proportions across grazing management and sward height treatments. During the trial, perennial ryegrass (L. perenne) was the dominant species in all the treatments comprising values always above the 0.50 proportion. In contrast, dead clover and other species as Festuca arundinacea, Holcus lanatus, Poa annua, Agrostis capillaris and Dactylis glomerata, and herbaceous weeds as Cerastium arvense, Anthoxanthum odoratum, Taraxacum officinale appeared very occasionally and were minor components ranging between 0.00 and 0.03 overall proportion.

The proportion of live white clover was the sward component most affected by the experiment. Over the whole experimental period, the mean proportion

Fig. 1. The vertical distribution of rye grass and clover components in each sward treatment and stock last species grazing management at the beginning $(7 \mathrm{March})$ and end (16 June) of the experiment. Data are expressed as number of point-quadrat hits per 100 loci in each $1-\mathrm{cm}$ horizon. 
of clover laminae-petiole increased $(P<0.001)$ across all management and height treatments with peak increases taking place from 7 March to 10 April. Stock species grazing had a significant effect on this sward clover appearance, so that by the end of the experiment, the average presence of live clover achieved higher proportions in those paddocks most recently grazed by goats than in those most recently grazed by sheep across the rotational and sequential treatments, with the mixed management having intermediate proportions. That clover enhancement due to goat last grazing was more marked on the sward upper layers. Also, the occurrences of dead and stem ryegrass material and of clover flower component were lower and higher, respectively, in the goat-last grazed paddocks than in the sheep-last grazed paddocks.

\subsection{Sheep performance}

Lamb and ewe performance data are shown for both genotypes in Tables 3 and 4, respectively. Lamb growth rates were significantly affected by the grazing management $(P<0.001)$, with much lower liveweight gains recorded per head in the rotational than in the sequential and mixed managements. These differences were much higher on lambs suckled from Lacha ewes: 147, 234, 195, 246 and $259 \mathrm{~g} \mathrm{LW} / \mathrm{d}$ $(\mathrm{SED}=12.8)$ than from Gallega ewes: 141, 169, 198, 198 and $179 \mathrm{~g} \mathrm{LW} / \mathrm{d}(\mathrm{SED}=11.7)$ in the rotational-low, sequential-high, sequential-low, mixed-high and mixed-low treatments, respectively. Similarly, the ewe's body condition and liveweight changes were affected significantly by the different grazing strategies imposed. Sheep daily liveweight gains were ranged over the whole lactation period from $77 \mathrm{~g}$ to $207 \mathrm{~g}$ and from $91 \mathrm{~g}$ to 181 $\mathrm{g}$ on Lacha and Gallega breeds, respectively, with the sequential management recording the highest weight gains within each height treatment.

\subsection{Stock productivity}

The product of animal grazing days (including tester plus replacement animals) and individual rates of liveweight gain gives output of liveweight gain per hectare and is shown in Table 5. There were contrasting sheep net liveweight gains per hectare between grazing managements with the mixed treatment having lower sheep productivity per hectare

Table 4

Liveweight and body condition score of the core lactating ewes from Lacha and Gallega genotypes at each sampling date grazing with goats ryegrass /clover sward height treatments from turnout (7 March) to lamb weaning (16 June)

\begin{tabular}{|c|c|c|c|c|c|c|c|c|c|}
\hline & \multirow[t]{4}{*}{ Date } & \multicolumn{8}{|c|}{ Grazing management } \\
\hline & & \multicolumn{2}{|c|}{ Rotational } & \multicolumn{3}{|c|}{ Sequential } & \multicolumn{3}{|l|}{ Mixed } \\
\hline & & \multicolumn{5}{|c|}{ Sward height } & \multirow[b]{2}{*}{ High } & \multirow[b]{2}{*}{ Low } & \multirow[b]{2}{*}{ SEM } \\
\hline & & Low & SE & High & Low & SEM & & & \\
\hline \multicolumn{10}{|l|}{$\overline{L a c h a}$} \\
\hline \multirow{4}{*}{ Liveweight (kg LW) } & 7 March & $32.1^{\mathrm{a}}$ & 0.64 & $31.8^{\mathrm{a}}$ & $31.6^{\mathrm{a}}$ & 0.44 & $32.0^{\mathrm{a}}$ & $32.3^{\mathrm{a}}$ & 0.50 \\
\hline & 10 April & $38.3^{\mathrm{a}}$ & 0.53 & $43.1^{\mathrm{b}}$ & $40.3^{c}$ & .047 & $39.1^{\mathrm{ac}}$ & $36.6^{\mathrm{d}}$ & 0.43 \\
\hline & 15 May & 42.4 & 0.62 & $47.4^{\mathrm{b}}$ & $43.4^{\mathrm{a}}$ & 0.39 & 43.7 & $38.9^{\mathrm{c}}$ & 0.53 \\
\hline & 16 June & $44.3^{\mathrm{a}}$ & 0.63 & $52.7^{\mathrm{b}}$ & $45.0^{\mathrm{ac}}$ & 0.48 & $46.0^{\mathrm{c}}$ & $40.1^{\mathrm{d}}$ & 0.47 \\
\hline Initial body condition (units) & & $2.25^{\mathrm{a}}$ & 0.125 & $2.23^{\mathrm{a}}$ & $2.20^{\mathrm{a}}$ & 0.101 & $2.26^{\mathrm{a}}$ & $2.19^{\mathrm{a}}$ & 0.139 \\
\hline Final body condition (units) & & $2.26^{\mathrm{a}}$ & 0.114 & $2.98^{\mathrm{b}}$ & $2.51^{\mathrm{c}}$ & 0.099 & $2.92^{\mathrm{b}}$ & $2.30^{\mathrm{a}}$ & 0.127 \\
\hline \multicolumn{10}{|l|}{ Gallega } \\
\hline \multirow[t]{4}{*}{ Liveweight (kg LW) } & 7 March & $28.3^{\mathrm{a}}$ & 0.42 & $28.4^{\mathrm{a}}$ & $28.7^{\mathrm{a}}$ & 0.22 & $28.5^{\mathrm{a}}$ & $28.1^{\mathrm{a}}$ & 0.32 \\
\hline & 10 April & $33.6^{\mathrm{a}}$ & 0.47 & $38.2^{\mathrm{b}}$ & $35.9^{\mathrm{c}}$ & 0.31 & $34.2^{\mathrm{a}}$ & $33.3^{\mathrm{a}}$ & 0.34 \\
\hline & 15 May & $36.2^{\mathrm{a}}$ & 0.52 & $42.9^{\mathrm{b}}$ & $38.2^{\mathrm{c}}$ & 0.29 & $36.7^{\mathrm{a}}$ & $35.4^{\mathrm{a}}$ & 0.38 \\
\hline & 16 June & $39.6^{\mathrm{a}}$ & 0.41 & $46.7^{\mathrm{b}}$ & $40.9^{c}$ & 0.40 & $38.0^{\mathrm{d}}$ & $37.3^{\mathrm{d}}$ & 0.31 \\
\hline Initial body condition (units) & & $2.27^{\mathrm{a}}$ & 0.134 & $2.22^{\mathrm{a}}$ & $2.19^{\mathrm{a}}$ & 0.109 & $2.23^{\mathrm{a}}$ & $2.21^{\mathrm{a}}$ & 0.137 \\
\hline Final body condition (units) & & $2.72^{\mathrm{ab}}$ & 0.127 & $2.91^{\mathrm{b}}$ & $2.81^{\mathrm{a}}$ & 0.103 & $2.60^{\mathrm{a}}$ & $2.56^{\mathrm{a}}$ & 0.141 \\
\hline
\end{tabular}

Means in the same row with different superscripts differ significantly $(P<0.05)$. 
Table 5

Grazing days, net liveweight gain and total output for the experimental herd (core plus replacement animals) across the different treatments

\begin{tabular}{|c|c|c|c|c|c|c|c|c|c|}
\hline & \multicolumn{9}{|c|}{ Grazing management } \\
\hline & \multicolumn{3}{|c|}{ Rotational } & \multicolumn{3}{|c|}{ Sequential } & \multicolumn{3}{|l|}{ Mixed } \\
\hline & \multicolumn{6}{|c|}{ Sward height } & \multirow[b]{2}{*}{ High } & \multirow[b]{2}{*}{ Low } & \multirow[b]{2}{*}{ SEM } \\
\hline & High & Low & SEM & High & Low & SEM & & & \\
\hline \multicolumn{10}{|l|}{$\overline{\text { Grazing days (days / ha) }}$} \\
\hline Ewe & - & $3219^{\mathrm{a}}$ & 89.5 & $1773^{\mathrm{b}}$ & $2546^{\mathrm{c}}$ & 63.1 & $1400^{\mathrm{d}}$ & $1700^{\mathrm{b}}$ & 73.7 \\
\hline Lamb & - & $2900^{\mathrm{b}}$ & 93.7 & $1700^{\mathrm{b}}$ & $2400^{c}$ & 59.8 & $1400^{\mathrm{d}}$ & $1700^{\mathrm{b}}$ & 78.3 \\
\hline Doe & $4498^{\mathrm{a}}$ & - & 125.3 & $2392^{b}$ & $3338^{c}$ & 98.8 & $1400^{\mathrm{e}}$ & $1700^{\mathrm{e}}$ & 113.2 \\
\hline \multicolumn{10}{|c|}{ Liveweight gain $(\mathrm{kg} \mathrm{LW} / \mathrm{ha})$} \\
\hline Ewe & - & $375^{\mathrm{a}}$ & 11.2 & $344^{\mathrm{b}}$ & $335^{\mathrm{b}}$ & 7.8 & $163^{\mathrm{c}}$ & $143^{\mathrm{c}}$ & 13.1 \\
\hline Lamb & - & $418^{\mathrm{a}}$ & 11.8 & $343^{\mathrm{b}}$ & $472^{c}$ & 8.8 & $311^{\mathrm{b}}$ & $372^{\mathrm{d}}$ & 13.0 \\
\hline Doe & $397^{\mathrm{a}}$ & - & 14.5 & $392^{\mathrm{a}}$ & $355^{\mathrm{b}}$ & 12.2 & $145^{\mathrm{c}}$ & $110^{\mathrm{c}}$ & 9.1 \\
\hline Total output (kg LW/ha) & $397^{\mathrm{a}}$ & $793^{\mathrm{b}}$ & $12.5^{*}$ & $1079^{c}$ & $1162^{d}$ & $9.6^{+}$ & $619^{\mathrm{e}}$ & $11.7^{+}$ & \\
\hline
\end{tabular}

Means in the same row with different superscripts differs significantly $(P<0.05)$.

${ }^{*}$ From a pooled standard error between height treatments within a management treatment.

${ }^{+}$From a pooled standard error of means between height treatments within a management treatment.

(495 kg LW/ha) than the rotational (793 kg LW/ha) and sequential treatments $(747 \mathrm{~kg} \mathrm{LW} / \mathrm{ha}$ ) (pooled SED $=75.3 ; \quad P<0.01)$. Additionally, goat output per hectare was the lowest in the mixed treatment. Since grazing days/ha for sheep and goats were reduced significantly in the high height treatments of the mixed and sequential managements, there was an overall extra benefit of 6 and $83 \mathrm{~kg} \mathrm{LW} / \mathrm{ha}$ in their corresponding low height treatments.

In relation to the stock health, core does carried by the end of the experiment more strongylate eggs per gram (e.p.g.) under mixed grazing than under rotational and sequential grazing (1800 vs. 1250 and 1200 e.p.g., respectively; SED $=145.2 ; P<0.001$ ). However, all groups of ewes had similar low egg counts (200 e.p.g.) independently of the grazing strategy.

\section{Discussion}

To avoid bias in determining sheep grazing behaviour within a flock, large paddocks containing at least 10 core ewes for each genotype were considered necessary for being representative (Penning et al., 1993). That increased demands on the available pasture of the experimental area, and treatment pad- docks were not able to replicate. However, the paddock component of variance was small compared with the sampling variance in our data, and treatment effects could be assumed, as suggested by Armstrong et al. (1995), to be uninfluenced by the experimental layout design.

\subsection{Sward composition}

Despite mean sward surface heights being maintained close to target heights over the whole grazing trial, the distribution of the height measurements was more skewed in the sequential treatment and in those paddocks last grazed by sheep. That contrasts with the results of del Pozo and Wright (1995), but agrees well with the findings of Nicol et al. (1987), who observed a greater patchiness in a ryegrass/white clover pasture grazed by sheep than by goats. Nicol and Collins (1990) suggested that goats may browse from the top of the sward, whereas sheep tend to graze deeper resulting, therefore, in a less evenly grazed sward. Thus, alternating through a sequential procedure, the timing of sheep and goat grazing with enough length can exacerbate more the height patchiness in a pasture than if swards are grazed rotationally or concurrently mixed. Furthermore, Betteridge et al. (1994) reported on temperate grass-clover 
swards that goats, when compared with sheep, left shorter heights from residual plant tillers because they grazed a larger proportion of the paddock each day. Also, differences in sward height heterogeneity due to species grazing was probably accounted for by the fact that goats seem to have greater capabilities than sheep to deal with reproductive and senescent material, and may graze them off at lower residual heights (Gurung et al., 1994). That also may explain the higher accumulation of dead and stem material that resulted through the experiment in the swards last grazed by sheep in comparison to swards last grazed by goats.

As reported by Thomson and Power (1993) on a mixed bull-goat grazing experiment and by Radcliffe et al. (1991) on a mixed sheep-goat grazing, the higher defoliation of long pasture and better elimination of pasture clumps by goats could maintain a better optimum height for canopy growth in swards grazed by goats than grazed by sheep. That could lead in the current trial to the outcome of higher herbage mass in those swards last grazed by goats. However, a binomial distribution of sward heights corresponding to 'frequently grazed' and 'infrequently grazed' components that Gibb and Ridout (1986) found under continuous cattle grazing, was not seen in this experiment because these effects, corroborating suggestions of Kitessa and Nicol (1996), appear to be less obvious under multispecies than monospecies grazing.

Throughout the grazing trial, there was heterogeneity both horizontally and vertically in the distribution of the grass and clover morphological components. Live clover tended to have a great proportion of its leaf area on the upper layers of the canopy, and that occurred markedly in the rotational and sequential managements. del Pozo et al. (1996) achieved increases until 0.37 clover proportion in grass / clover swards grazed by lambs after being previously grazed by goats, whereas Bown et al. (1989) also showed extra clover growths when pastures were grazed rotationally by a mob of goats, sheep and cattle. Thus, different timings of sheep and goat grazings seemed to facilitate more the clover outcome through the sward upper layers than mixed species management.

Furthermore, swards last grazed by goats tended to contain higher clover and less stem and dead proportions than swards last grazed by sheep, and that was accompanied by very marked visual differences. As suggested by Gong et al. (1993), these conflicting findings on sward clover content may result from the interaction of the feeding style of goats and sheep, with the position of clover within the vegetation strata relative to the grazing horizon of the animal. Goats, unlike sheep, seemed to not actively select Trifolium species particularly in the lower horizons, making these differences more dramatic on the sward surface (Fig. 1). That is in agreement with other results of Nicol and Collins (1990) and del Pozo et al. (unpublished) for sheep and goat cograzing, and of Milne et al. (1982) with sheep solely grazing. These authors showed that the composition of the diet in both sheep and goats were more closely related to changes in the composition of the upper layers of the sward than with the proportion of clover in the sward as a whole. However, these results cannot show that variability in diet clover is entirely explained by passive selection as Norton et al. (1990) showed in sheep, cattle and goats that their clover consumption might also differ within a season grazing.

\subsection{Animal responses}

Although herbage mass on offer to the experimental herd was higher in the goat-grazed swards, this is unlikely to be the major factor involved in the improvement of sheep liveweight gains with goat cograzing, as total herbage mass levels were always on this trial above the critical height levels recommended by Maxwell and Treacher (1987) for no limit in sheep weight gains. In accordance with the findings of Merchant and Riach (1994), the proportion of green leaf of ryegrass and clover components was not related in the current study to the different sward height treatments, since a relatively vegetative leafy state was maintained during most parts of the experiment. Therefore, the low appearance of grass seedheads and stems observed to occur through the experiment associated with increases of the live clover proportion appeared to influence more tangibly the lamb and ewe performances occurring in all treatments. Thus, the results obtained in sheep liveweight replenishments were much higher in this experiment than those achieved by Martinez and 
Osoro (1995) with similar local breeds and management conditions on a continuous mono-grazing system. In their experiment, sward surface heights were maintained between the $5.5-6.0 \mathrm{~cm}$ range, and lambs and ewes reached gains of 170-180 and 30-40 g $\mathrm{LW} / \mathrm{d}$ respectively. Also, when sward height was allowed to increase to $7.0-7.5 \mathrm{~cm}$ from the preceding spring period, they only managed to record gains of 195 vs. $220 \mathrm{~g} \mathrm{LW} / \mathrm{d}$ in lambs and 65 vs. $85 \mathrm{~g} / \mathrm{d}$ in ewes from Gallega and Lacha breeds, respectively. Similarly, Radcliffe et al. (1991) reported that pre-weaning lambs grazing simultaneously with goats in a 1:1 mixture had daily liveweight gains some $28 \%$ greater than those grazing sheep-only pastures. Also, Townsend and Radcliffe (1990) showed extra lamb growth rates before weaning on a sheep-goat mixed than on a sheep solely management. Since Ulyatt (1981) postulated that clover can promote better live-weight performances in lambs and Gibb and Treacher (1983) showed that increasing the ratio of white clover to perennial ryegrass in the diet of lactating ewes seemed to increase the growth rate of their lambs during lactation without having negative effects on the rate of depletion of the ewe's body reserves, it seems reasonable to think that ewes and lambs would perform better during their lactation period when grazing grass /clover swards with goats than solely managed. However, an adequate prophylactic treatment needs to be given to both stock species, as they might be susceptible to the same internal parasites.

Finally, Tables 3 and 4 show that higher body weight replenishments occurred at the high height treatments in sheep from Lacha than from Gallega genotypes, although those differences were reduced at the low height treatments. That was also previously reported by the study of Martinez and Osoro (1995), as they found that the mean sward height at which liveweight performance was maximized in these genotypes, was higher in ewes and lambs from Lacha than from Gallega breed. As postulated by Illius and Gordon (1990), the value of alternative food patches in the sward may depend not only on the proportion of the sward, but on the size of the grazer. Then, the interaction between sheep size and sward canopy structure might also have an important practical implication on the management of sheep when grazing grass/clover swards.

\section{Conclusions}

These results indicate quite different opportunities for goats to complement sheep when grazing together grass /clover swards. Goat's cograzing can be used to improve the clover content of pastures and benefit sheep lactation performances following different or concurrent grazing procedures with the rotational and sequential managements, resulting in higher sheep productivity per hectare than the mixed management. However, the choice of an adequate grazing management to integrate goats with sheep is flexible and diverse through season, and further interpretation of the results to achieve more efficient management systems will only be possible once information is also available regarding diet selected, herbage intake and animal health.

\section{Acknowledgements}

The authors gratefully acknowledge the assistance of Dr. L. Ortega and Mr. J. Requejo for parasitology analysis, Mr. J.J. Ormazábal for stock work, and Mrs. E. López for the preparation of the tables. Dr. M. del Pozo was funded by the MEC of Spain.

\section{References}

Armstrong, R.H., Robertson, E.H., Hunter, E.A., 1995. The effect of sward height and its direction of change on the herbage intake, diet selection and performance of weaned lambs grazing ryegrass swards. Grass Forage Sci. 50, 389-398.

Barthram, G.T., 1986. Experimental techniques: the HFRO sward stick. In: HFRO Biennial Report, 1984-1985, pp. 29-30.

Betteridge, K., Fletcher, R., Liu, Y., Costall, D.A., Devantier, B.P., 1994. Rate of removal of grass from mixed pastures by cattle, sheep and goat grazing. Proc. N.Z. Grassl. Assoc. 56, 61-65.

Bown, M.D., McCall, D.G., Scott, M.L., Watson, T.G., Dow, B.W., 1989. The effect of integrated grazing of goats, sheep and cattle on animal productivity and health on high-producing hill country pastures. Proc. N.Z. Soc. Anim. Prod. 49, $165-169$.

Clark, D.A., Lambert, M.G., Rolston, M.P., Dymock, N., 1982. Diet selection by goats and sheep on hill country. Proc. N.Z. Soc. Anim. Prod. 42, 155-157.

Collins, W.B., 1989. Single and mixed grazing of cattle, sheep and goats. PhD Thesis. Univ. of Lincoln, New Zealand, 195 pp. 
del Pozo, M., Wright, I., 1995. Integration of sheep and goats in grazing systems on grass / clover swards. In: Laker, J.P., Russel, A.J.F. (Eds.), The Nutrition and Grazing Ecology of Specialty Fibre Producing Animals. Eur. Fine Fibre Network, Occas. Publi., Vol. 3, pp. 151-162.

del Pozo, M., Wright, I., Whyte, T.K., Colgrove, P.M., 1996. Effects of grazing by sheep or goats on sward composition in ryegrass/white clover pasture and on subsequent performance of weaned lambs. Grass Forage Sci. 51, 142-154.

Gibb, M.J., Ridout, M.S., 1986. The fitting of frequency distributions to height measurements of sward height. Grass Forage Sci. 41, 247-249.

Gibb, M.J., Treacher, T.T., 1983. The performance of lactating ewes offered diets containing different proportions of fresh perennial ryegrass and white clover. Anim. Prod. 37, 433-440.

Gong, Y., Hodgson, J., Lambert, M.G., Chu, A.C.P., Gordon, I.L., 1993. Comparison of bite weight and bite dimensions of sheep and goats grazing a range of grasses and clovers. Proc. XVIIth Int. Grassl. Cong., Hamilton, New Zealand, pp. 726-728.

Grant, S.A., Bolton, G.R., Russel, A.J.F., 1984. The utilization of sown and indigenous plant species by sheep and goats grazing hill pastures. Grass Forage Sci. 39, 361-370.

Gurung, N.K., Jallow, O.A., McGregor, B.A., Watson, M.J., McIlroy, B.K.M.H., Holmes, J.H.G., 1994. Complementary selection and intake of annual pastures by sheep and goats. Small Rumin. Res. 14, 185-192.

Illius, A.W., Gordon, I.J., 1990. Constraints on diet selection and foraging behaviour in mammalian herbivores. In: Hughes, R.N. (Ed.), Behavioural Mechanisms of Food Selection, pp. 369-392.

Kitessa, S.M., Nicol, A.M., 1996. Frequency distribution of sward height on pastures grazed by cattle alone or co-grazed with sheep. N.Z. Soc. Anim. Prod. 56th Annual Conf., p. 46.

Martinez, A., Osoro, K., 1995. Respuesta del ganado ovino a la altura de la hierba disponible en pastos de raigrás y trébol. [Ovine responses to available sward heights in ryegrass /clover pastures] ITEA 16, 171-173.

Maxwell, T.J., Treacher, T.T., 1987. Decision rules for grassland management. In: Pollott, G.E. (Ed.), Efficient Sheep Production from Grass. Br. Grassland Soc., pp. 67-78.

Merchant, M., Riach, D.J., 1994. The intake and performance of cashmere goats grazing sown swards. Grass Forage Sci. 49, 429-437.

Milne, J.A., Hodgson, J., Thompson, R., Souter, W.G., Barthram, G.T., 1982. The diet ingested by sheep grazing swards differ- ing in white clover and perennial ryegrass content. Grass Forage Sci. 37, 209-218.

Nicol, A.M., Collins, H.A., 1990. Estimation of the pasture horizons grazed by cattle, sheep and goats during single and mixed grazing. Proc. N.Z. Soc. Anim. Prod. 50, 49-53.

Nicol, A.M., Poppi, D.P., Alan, M.R., Collins, H.A., 1987. Dietary differences between sheep and goats. Proc. N.Z. Grassl. Assoc. 48, 199-205.

Norton, B.W., Kennedy, P.J., Hales, J.W., 1990. Grazing management studies with Australian cashmere goats: 3. Effect of season on the selection of diet by cattle, sheep and goats from two tropical grass-legume pastures. Aust. J. Exp. Agric. 30, 783-788.

Osoro, K., Martinez, A., 1995. Grazing behaviour and performance of goats and sheep on natural and improved vegetation. In: Laker, J.P., Russel, A.J.F. (Eds.), The Nutrition and Grazing Ecology of Specialty Fibre Producing Animals. Eur. Fine Fibre Network, Occas. Publi., Vol. 3, pp. 109-123.

Penning, P.D., Johnson, R.H., Orr, R.J., 1996. Effects of continuous stocking with sheep or goats on sward composition and animal production from a grass and white clover pasture. Small Rumin. Res. 21, 19-29.

Penning, P.D., Parsons, A.J., Newman, J.A., Orr, R.J., Harvey, A., 1993. The effects of group size on grazing in sheep. Appl. Anim. Behav. Sci. 37, 101-109.

Radcliffe, J.E., Townsend, R.J., Baird, D.B., 1991. Mixed and separate grazing of sheep and goats at two stocking rates. N.Z. J. Agric. Res. 34, 167-176.

Rhodes, I., Collins, R.P., 1993. Canopy structure. In: Davies, A., Baker, R.D., Grant, S., Laidlaw, A.S. (Eds.), Sward Measurement Handbook. Br. Grassland Soc., pp. 139-156.

Russel, A.J.F., Doney, J.M., Gunn, R.G., 1969. Subjective assessment of body fat in live sheep. J. Agric. Sci. 72, 451-454.

Russel, A.J.F., Maxwell, T.J., Bolton, G.R., Currie, D.C., White, I.R., 1983. A note on the possible use of goats in hill country sheep grazing systems. Anim. Prod. 36, 313-316.

Thomson, N.A., Power, M.T., 1993. An evaluation of a possible complementary effect of grazing goats with bulls in an intensive bull beef system. Proc. N.Z. Soc. Anim. Prod. 53, 23-27.

Townsend, R.J., Radcliffe, J.E., 1990. Lamb growth rates improve as goat to sheep ratio increases. Proc. N.Z. Grassl. Assoc. 52 , $115-118$.

Ulyatt, M.J., 1981. The feeding value of temperature pastures. In: Morley, F.H.W. (Ed.), Grazing Animals, World Animal Science, Vol. 1B. Elsevier, Amsterdam, pp. 125-141. 